\title{
Existence and stability results for nonlocal initial value problems for differential equations with Hilfer fractional derivative
}

\author{
Mouffak Benchohra, Soufyane Bouriah and Juan J. Nieto
}

\begin{abstract}
In this paper, we establish sufficient conditions for the existence and stability of solutions for a class of nonlocal initial value problems for differential equations with Hilfer's fractional derivative, The arguments are based upon the Banach contraction principle. Two examples are included to show the applicability of our results.
\end{abstract}

Mathematics Subject Classification (2010): 26A33.

Keywords: Hilfer's fractional derivative, Caputo and Riemann-Liouville sense, existence, uniqueness, fixed point, Ulam stability.

\section{Introduction}

In our paper, we study the following nonlocal initial value problem

$$
\begin{gathered}
D_{0^{+}}^{\alpha, \beta} y(t)=f\left(t, y(t), D_{0^{+}}^{\alpha, \beta} y(t)\right), \quad \text { for every } t \in(0, T], T>0, \\
I_{0^{+}}^{1-\gamma} y\left(0^{+}\right)=\sum_{i=1}^{m} \lambda_{i} y\left(\tau_{i}\right), \quad \tau_{i} \in(0, T],
\end{gathered}
$$

where $0<\alpha<1,0 \leq \beta \leq 1, \gamma=\alpha+\beta-\alpha \beta, f:(0, T] \times \mathbb{R} \times \mathbb{R} \longrightarrow \mathbb{R}, \tau_{i}, i=1,2, \ldots, m$ are pre-fixed points satisfying $0<\tau_{1} \leq \cdots \leq \tau_{m}<T, \lambda_{i}$ are real numbers and

$$
\sum_{i=1}^{m} \lambda_{i} \tau_{i}^{\gamma-1} \neq \Gamma(\gamma),
$$

$D_{0^{+}}^{\alpha, \beta}$ denotes the generalized Riemann-Liouville derivative operator introduced by Hilfer in [7].

In the recent years, fractional calculus has gained much interest mainly thanks to the increasing presence of research works in the applied sciences considering models based on fractional operators see for example $[1,2,6,9,12,16,17,21]$. Beside that, the 
mathematical study of fractional calculus has proceeded, leading to intersections with other mathematical fields such as probability and the study of stochastic processes. In the literature, several different definitions of fractional integrals and derivatives are present. Some of them such as the Riemann-Liouville integral, the Caputo and the Riemann-Liouville derivatives are thoroughly studied and actually used in applied models. Hilfer has introduced a generalized form of the Riemann-Liouville fractional derivative [7]. In short, Hilfer fractional derivative $D_{0^{+}}^{\alpha, \beta}$ is an interpolation between the Riemann-Liouville and Caputo fractional derivatives, see [10, 13, 17, 19]. It has many applications in fractional evolutions equations [8], and physical problems [15]. Also in the theoretical simulation of dielectric relaxation in glass forming materials. In [4], Furati et al. considered an initial value problem for a class of nonlinear fractional differential equations involving Hilfer fractional derivative. In [3], the authors consider the Ulam stability for nonlinear implicit fractional differential equations with Hadamard derivative. In [18], the solution of a fractional diffusion equation with a Hilfer time fractional derivative was obtained in terms of Mittag-Leffler functions and Fox's H-function. To the best of our knowledge, there has no results about the stability of differential equations with Hilfer fractional derivative.

Motivated by the works [3, 19], we prove in this paper the existence, uniqueness and stability for the non-linear nonlocal problem (1.1)-(1.2) in a weighted space of continuous functions. The present work is organized as follows. In Section 2, some notations are introduced and we recall some concepts of preliminaries about fractional calculus and auxiliary results. The proof for the main results is presented in Section 3 by applying the Banach fixed point theorem. In Section 4, the Ulam stability of our problem will be study. Finally, in the last section, we give two examples to illustrate the applicability of our main results.

\section{Preliminaries}

In this section, we recall some basic definitions and results concerning the fractional calculus, that we will use in the next sections .

Let $J:=[0, T]$. By $C(J), A C(J)$ and $C^{n}(J)$ we denote the spaces of continuous, absolutely continuous and $n$ times continuously differentiable functions on $J$, respectively. We denote by $L^{p}(J), p \geq 0$, the space of Lebesgue integrable functions on $J$.

We consider the weighted spaces of continuous functions

$$
\begin{gathered}
C_{\gamma}(J)=\left\{y:(0, T] \rightarrow \mathbb{R}: t^{\gamma} y(t) \in C(J)\right\}, 0 \leq \gamma<1, \\
C_{\gamma}^{n}(J)=\left\{y \in C^{n-1}(J): y^{(n)} \in C_{\gamma}(J)\right\}, n \in \mathbb{N}, \\
C_{\gamma}^{0}(J)=C_{\gamma}(J),
\end{gathered}
$$

with the norms

$$
\|y\|_{C_{\gamma}}=\left\|t^{\gamma} y(t)\right\|_{\infty}=\sup _{t \in J}\left|t^{\gamma} y(t)\right|
$$


and

$$
\|y\|_{C_{\gamma}^{n}}=\sum_{k=0}^{n-1}\left\|y^{(k)}\right\|_{\infty}+\left\|y^{(n)}\right\|_{C_{\gamma}} .
$$

These spaces satisfy the following properties.

- $C_{0}(J)=C(J)$.

- $C_{\gamma}^{n}(J) \subset A C^{n}(J)$.

- $C_{\gamma_{1}}(J) \subset C_{\gamma_{2}}(J), 0 \leq \gamma_{1}<\gamma_{2}<1$.

Definition 2.1. ([10, 13]). The fractional (arbitrary) order integral of the function $h \in L^{1}\left([0, T], \mathbb{R}_{+}\right)$of order $\alpha \in \mathbb{R}_{+}$called the left-sided Riemann-Liouville integral of the function $h$ is defined by

$$
\left(I_{0^{+}}^{\alpha} h\right)(t)=\frac{1}{\Gamma(\alpha)} \int_{0}^{t}(t-s)^{\alpha-1} h(s) d s,
$$

where $\Gamma$ is the Euler gamma function defined by $\Gamma(\alpha)=\int_{0}^{\infty} t^{\alpha-1} e^{-t} d t, \alpha>0$.

The following lemmas provide some mapping properties of $I_{0^{+}}^{\alpha}$. The proofs can be found in [11].

Lemma 2.2. For $\alpha>0, I_{0^{+}}^{\alpha}$ maps $C(J)$ into $C(J)$.

Lemma 2.3. Let $\alpha>0$ and $0 \leq \gamma<1$. Then, $I_{0^{+}}^{\alpha}$ is bounded from $C_{\gamma}(J)$ into $C_{\gamma}(J)$

Lemma 2.4. Let $\alpha>0$ and $0 \leq \gamma<1$. If $\gamma \leq \alpha$, then $I_{0^{+}}^{\alpha}$ is bounded from $C_{\gamma}(J)$ into $C(J)$.

Lemma 2.5. [4] Let $0 \leq \gamma<1$ and $y \in C_{\gamma}(J)$. Then

$$
I_{0^{+}}^{\alpha} y(0):=\lim _{t \rightarrow 0^{+}} I_{0^{+}}^{\alpha} y(t)=0, \quad 0 \leq \gamma<\alpha .
$$

Definition 2.6. [5] The Riemann-Liouville left-sided fractional derivative $D_{0^{+}}^{\alpha}$ of order $\alpha$ is defined by

$$
\left(D_{0^{+}}^{\alpha} y\right)(t)=D\left(I_{0^{+}}^{1-\alpha} y\right)(t), \quad\left(t>0,0<\alpha<1, D=\frac{d}{d t}\right)
$$

that is

$$
\left(D_{0^{+}}^{\alpha} y\right)(t)=\frac{1}{\Gamma(1-\alpha)} \frac{d}{d t} \int_{0}^{t}(t-s)^{\alpha} y(s) d s,
$$

when $\alpha=1$ we have $\left(D_{0^{+}}^{\alpha} y\right)=D y$. In particular, when $\alpha=0,\left(D_{0^{+}}^{0} y\right)=y$.

Lemma 2.7. [4] For $t>0$, we have

$$
\begin{aligned}
& {\left[I_{0^{+}}^{\alpha} t^{\beta-1}\right](t)=\frac{\Gamma(\beta)}{\Gamma(\alpha+\beta)} t^{\beta+\alpha-1}, \quad \alpha \geq 0, \beta>0 .} \\
& {\left[D_{0^{+}}^{\alpha} t^{\alpha-1}\right](t)=0, \quad 0<\alpha<1 .}
\end{aligned}
$$

The following lemmas follows by direct calculations using Dirichlet formula 
Lemma 2.8. Let $\alpha>0, \beta>0$ and $y \in L^{1}(J)$. Then

$$
I_{0^{+}}^{\alpha} I_{0^{+}}^{\beta} y(t)=I_{0^{+}}^{\alpha+\beta} y(t), \text { a.e. } t \in J .
$$

In particular, if $y \in C_{\gamma}(J)$ or $y \in C(J)$, then equality holds at every $t \in(0, T]$ or $t \in[0, T]$, respectively.

Lemma 2.9. Let $\alpha>0,0 \leq \gamma<1$ and $y \in C_{\gamma}(J)$. Then

$$
D_{0^{+}}^{\alpha} I_{0^{+}}^{\alpha} y(t)=y(t), \text { for all } t \in(0, T] \text {. }
$$

Lemma 2.10. [17] Let $0<\alpha<1,0 \leq \gamma<1$. If $y \in C_{\gamma}(J)$ and $I_{0^{+}}^{1-\alpha} y \in C_{\gamma}^{1}(J)$, then

$$
I_{0^{+}}^{\alpha} D_{0^{+}}^{\alpha} y(t)=y(t)-\frac{I_{0^{+}}^{1-\alpha} y(0)}{\Gamma(\alpha)} t^{\alpha-1}, \text { for all } t \in(0, T] \text {. }
$$

Let $\alpha \in(0,1), \beta \in[0,1]$ and $y \in L^{1}\left(J, \mathbb{R}^{n}\right)$. We say that the function $y$ possesses the left-sided generalized Riemann-Liouville derivative (so called Hilfer derivative) $D_{0^{+}}^{\alpha, \beta}$ of order $\alpha$ and type $\beta$, if the function $I_{0^{+}}^{(1-\alpha)(1-\beta)} y$ is absolutely continuous on $J$ and then

$$
\left(D_{0^{+}}^{\alpha, \beta} y\right)(t):=\left(I_{0^{+}}^{\beta(1-\alpha)} D I_{0^{+}}^{(1-\alpha)(1-\beta)} y\right)(t), \text { a.e. } t \in J
$$

The operator $D_{0^{+}}^{\alpha, \beta} y$, given by (2.1), was introduced by Hilfer in [7].

Remark 2.11. [4]

1. The Hilfer derivative $D_{0^{+}}^{\alpha, \beta} y$ can be written as

$$
\left(D_{0^{+}}^{\alpha, \beta} y\right)(t):=\left(I_{0^{+}}^{\beta(1-\alpha)} D I_{0^{+}}^{(1-\gamma)} y\right)(t)=\left(I_{0^{+}}^{\beta(1-\alpha)} D_{0^{+}}^{\gamma} y\right)(t)=\left(I_{0^{+}}^{\gamma-\alpha} D_{0^{+}}^{\gamma} y\right)(t)
$$

for a.e. $t \in J$, where $\gamma=\alpha+\beta-\alpha \beta$.

2. The $D_{0^{+}}^{\alpha, \beta} y$ derivative is considered as an interpolator between the RiemannLiouville and Caputo derivative since

$$
D_{0^{+}}^{\alpha, \beta} y= \begin{cases}D_{0^{+}}^{\alpha} y, & \beta=0 \\ { }^{C} D_{0^{+}}^{\alpha} y, & \beta=1 .\end{cases}
$$

3. The parameter $\gamma$ satisfies

$$
0<\gamma \leq 1, \quad \gamma \geq \alpha, \quad \gamma>\beta, \quad 1-\gamma<1-\beta(1-\alpha)
$$

We introduce the spaces

$$
C_{1-\gamma}^{\alpha, \beta}(J)=\left\{y \in C_{1-\gamma}(J), D_{0^{+}}^{\alpha, \beta} y \in C_{1-\gamma}(J)\right\}
$$

and

$$
C_{1-\gamma}^{\gamma}(J)=\left\{y \in C_{1-\gamma}(J), D_{0^{+}}^{\gamma} y \in C_{1-\gamma}(J)\right\} .
$$

Since $D_{0^{+}}^{\alpha, \beta} y=I_{0^{+}}^{\beta(1-\alpha)} D_{0^{+}}^{\gamma} y$, it follows from Lemma 2.3 that

$$
C_{1-\gamma}^{\gamma}(J) \subset C_{1-\gamma}^{\alpha, \beta}(J) \subset C_{1-\gamma}(J)
$$

The following lemma follows directly from semigroup property in Lemma 2.8. 
Lemma 2.12. Let $0<\alpha<1,0 \leq \beta \leq 1$, and $\gamma=\alpha+\beta-\alpha \beta$. If $y \in C_{1-\gamma}^{\gamma}(J)$, then

$$
I_{0^{+}}^{\gamma} D_{0^{+}}^{\gamma} y=I_{0^{+}}^{\alpha} D_{0^{+}}^{\alpha, \beta} y
$$

and

$$
D_{0^{+}}^{\gamma} I_{0^{+}}^{\alpha} y=D_{0^{+}}^{\beta(1-\alpha)} y
$$

For the proof of the following lemmas, we can see [4].

Lemma 2.13. Let $y \in L^{1}(J)$. If $D_{0^{+}}^{\beta(1-\alpha)} y$ exists and in $L^{1}(J)$ then

$$
D_{0^{+}}^{\alpha, \beta} I_{0^{+}}^{\alpha} y=I_{0^{+}}^{\beta(1-\alpha)} D_{0^{+}}^{\beta(1-\alpha)} y .
$$

Lemma 2.14. Let $0<\alpha<1,0 \leq \beta \leq 1$, and $\gamma=\alpha+\beta-\alpha \beta$. If $y \in C_{1-\gamma}(J)$ and $I_{0^{+}}^{1-\beta(1-\alpha)} y \in C_{1-\gamma}^{1}(J)$ then $D_{0^{+}}^{\alpha, \beta} I_{0^{+}}^{\alpha} y$ exists in $(0, T]$ and

$$
D_{0^{+}}^{\alpha, \beta} I_{0^{+}}^{\alpha} y(t)=y(t), \quad t \in(0, T] .
$$

Lemma 2.15. ([20]) Let $v:[0, T] \longrightarrow[0,+\infty)$ be a real function and $\omega(\cdot)$ is a nonnegative, locally integrable function on $[0, T]$. Assume that there are constants $a>0$ and $0<\alpha \leq 1$ such that

$$
v(t) \leq \omega(t)+a \int_{0}^{t}(t-s)^{-\alpha} v(s) d s,
$$

then, there exists a constant $K=K(\alpha)$ such that

$$
v(t) \leq \omega(t)+K a \int_{0}^{t}(t-s)^{-\alpha} \omega(s) d s, \text { for every } t \in[0, T] .
$$

For the implicit fractional-order differential equation (1.1), we adopt the definition in Rus ([14]) for Ulam-Hyers stability, generalized Ulam-Hyers stability, Ulam-HyersRassias stability and generalized Ulam-Hyers-Rassias stability.

Definition 2.16. The equation (1.1) is Ulam-Hyers stable if there exists a real number $c_{f}>0$ such that for each $\epsilon>0$ and for each solution $z \in C_{1-\gamma}^{\gamma}(J)$, of the inequality

$$
\left|D_{0^{+}}^{\alpha, \beta} z(t)-f\left(t, z(t), D_{0^{+}}^{\alpha, \beta} z(t)\right)\right| \leq \epsilon, t \in(0, T],
$$

there exists a solution $y \in C_{1-\gamma}^{\gamma}(J)$ of equation (1.1) with

$$
|z(t)-y(t)| \leq c_{f} \epsilon, t \in(0, T] .
$$

Definition 2.17. The equation (1.1) is generalized Ulam-Hyers stable if there exists $\psi_{f} \in C\left(\mathbb{R}_{+}, \mathbb{R}_{+}\right), \psi_{f}(0)=0$, such that for each solution $z \in C_{1-\gamma}^{\gamma}(J)$ of the inequality

$$
\left|D_{0^{+}}^{\alpha, \beta} z(t)-f\left(t, z(t), D_{0^{+}}^{\alpha, \beta} z(t)\right)\right| \leq \epsilon, t \in(0, T],
$$

there exists a solution $y \in C_{1-\gamma}^{\gamma}(J)$ of the equation (1.1) with

$$
|z(t)-y(t)| \leq \psi_{f}(\epsilon), t \in(0, T] .
$$


Definition 2.18. The equation (1.1) is Ulam-Hyers-Rassias stable with respect to $\varphi \in$ $C\left(J, \mathbb{R}_{+}\right)$if there exists a real number $c_{f}>0$ such that for each $\epsilon>0$ and for each solution $z \in C_{1-\gamma}^{\gamma}(J)$ of the inequality

$$
\left|D_{0^{+}}^{\alpha, \beta} z(t)-f\left(t, z(t), D_{0^{+}}^{\alpha, \beta} z(t)\right)\right| \leq \epsilon \varphi(t), t \in(0, T],
$$

there exists a solution $y \in C_{1-\gamma}^{\gamma}(J)$ of equation (1.1) with

$$
|z(t)-y(t)| \leq c_{f} \epsilon \varphi(t), t \in(0, T] .
$$

Definition 2.19. The equation (1.1) is generalized Ulam-Hyers-Rassias stable with respect to $\varphi \in C\left(J, \mathbb{R}_{+}\right)$if there exists a real number $c_{f, \varphi}>0$ such that for each solution $z \in C_{1-\gamma}^{\gamma}(J)$ of the inequality

$$
\left|D_{0^{+}}^{\alpha, \beta} z(t)-f\left(t, z(t), D_{0^{+}}^{\alpha, \beta} z(t)\right)\right| \leq \varphi(t), t \in(0, T],
$$

there exists a solution $y \in C_{1-\gamma}^{\gamma}(J)$ of equation (1.1) with

$$
|z(t)-y(t)| \leq c_{f, \varphi} \varphi(t), t \in(0, T] .
$$

Remark 2.20. A function $z \in C_{1-\gamma}^{\gamma}(J)$ is a solution of the inequality

$$
\left|D_{0^{+}}^{\alpha, \beta} z(t)-f\left(t, z(t), D_{0^{+}}^{\alpha, \beta} z(t)\right)\right| \leq \epsilon, t \in(0, T],
$$

if and only if there exists a function $\phi \in C_{1-\gamma}^{\gamma}(J)$ (which depends on solution $y$ ) such that

i). $|\phi(t)| \leq \epsilon, t \in(0, T]$.

ii). $D_{0^{+}}^{\alpha, \beta} z(t)=f\left(t, z(t), D_{0^{+}}^{\alpha, \beta} z(t)\right)+\phi(t), t \in(0, T]$.

Remark 2.21. Clearly,

i). Definition (2.6) $\Rightarrow$ Definition (2.7)

ii). Definition (2.8) $\Rightarrow$ Definition (2.9).

Remark 2.22. A solution of the implicit differential inequality

$$
\left|D_{0^{+}}^{\alpha, \beta} z(t)-f\left(t, z(t), D_{0^{+}}^{\alpha, \beta} z(t)\right)\right| \leq \epsilon, t \in(0, T],
$$

with fractional order is called an fractional $\epsilon$ - solution of the implicit fractional differential equation (1.1).

\section{Existence of solutions}

Let $\gamma=\alpha+\beta-\alpha \beta$ where $0<\alpha<1$ and $0 \leq \beta \leq 1$, let $f:(0, T] \times \mathbb{R} \times \mathbb{R} \rightarrow \mathbb{R}$ be a function such that $f(\cdot, y(\cdot), u(\cdot)) \in C_{1-\gamma}(J)$ for any $y, u \in C_{1-\gamma}(J)$ and let the operator $N: C_{1-\gamma}(J) \rightarrow C_{1-\gamma}(J)$ defined by

$$
N y(t)=w(t)+\frac{1}{\Gamma(\alpha)} \int_{0}^{t}(t-s)^{\alpha-1} g(s) d s, \quad t \in(0, T]
$$

where

$$
w(t)=\frac{t^{\gamma-1}}{\Gamma(\alpha)\left(\Gamma(\gamma)-\sum_{i=1}^{m} \lambda_{i} \tau_{i}^{\gamma-1}\right)} \sum_{i=1}^{m} \lambda_{i} \int_{0}^{\tau_{i}}\left(\tau_{i}-s\right)^{\alpha-1} g(s) d s
$$


and $g:(0, T] \rightarrow \mathbb{R}$ be a function satisfies the functional equation

$$
g(t)=f(t, y(t), g(t)) .
$$

Clearly, $w \in C_{1-\gamma}(J)$ and $g \in C_{1-\gamma}(J)$. Also, by Lemma 2.3, $N y \in C_{1-\gamma}(J)$.

Theorem 3.1. If $y \in C_{1-\gamma}^{\gamma}(J)$, then $y$ satisfies the problem (1.1) - (1.2) if and only if $y$ is the fixed point of operator $N$.

Proof. First, we prove the necessity. Let $y \in C_{1-\gamma}^{\gamma}(J)$ be a solution of problem (1.1) (1.2). We want to prove that $y$ is a fixed point of $N$. By the definition of $C_{1-\gamma}^{\gamma}(J)$, Lemma 2.4 and Definition 2.6, we have

$$
I_{0^{+}}^{1-\gamma} y \in C(J) \text { and } D_{0^{+}}^{\gamma} y=D\left(I_{0^{+}}^{1-\gamma} y\right) \in C_{1-\gamma}(J) .
$$

Thus, we have

$$
I_{0^{+}}^{1-\gamma} y \in C_{1-\gamma}^{1}(J)
$$

Now, applying Lemma 2.10 to obtain

$$
I_{0^{+}}^{\gamma} D_{0^{+}}^{\gamma} y(t)=y(t)-\frac{I_{0^{+}}^{1-\gamma} y\left(0^{+}\right)}{\Gamma(\gamma)} t^{\gamma-1}, \quad t \in(0, T] .
$$

Since $D_{0^{+}}^{\gamma} y \in C_{1-\gamma}(J)$, Lemma 2.12 yields

$$
\left(I_{0^{+}}^{\gamma} D_{0^{+}}^{\gamma} y\right)(t)=\left(I_{0^{+}}^{\alpha} D_{0^{+}}^{\alpha, \beta} y\right)(t)=I_{0^{+}}^{\alpha} g(t), \quad t \in(0, T]
$$

From (3.2) and (3.3), we obtain

$$
y(t)=\frac{I_{0^{+}}^{1-\gamma} y\left(0^{+}\right)}{\Gamma(\gamma)} t^{\gamma-1}+\frac{1}{\Gamma(\alpha)} \int_{0}^{t}(t-s)^{\alpha-1} g(s) d s, \quad t \in(0, T] .
$$

Next, we substitute $t=\tau_{i}$ into the above equation

$$
y\left(\tau_{i}\right)=\frac{I_{0^{+}}^{1-\gamma} y\left(0^{+}\right)}{\Gamma(\gamma)} \tau_{i}^{\gamma-1}+\frac{1}{\Gamma(\alpha)} \int_{0}^{\tau_{i}}\left(\tau_{i}-s\right)^{\alpha-1} g(s) d s, \quad t \in(0, T],
$$

by multiplying $\lambda_{i}$ to both sides of (3.5), we can write

$$
\lambda_{i} y\left(\tau_{i}\right)=\frac{I_{0^{+}}^{1-\gamma} y\left(0^{+}\right)}{\Gamma(\gamma)} \lambda_{i} \tau_{i}^{\gamma-1}+\frac{\lambda_{i}}{\Gamma(\alpha)} \int_{0}^{\tau_{i}}\left(\tau_{i}-s\right)^{\alpha-1} g(s) d s .
$$

Thus, we have

$$
\begin{aligned}
I_{0^{+}}^{1-\gamma} y\left(0^{+}\right) & =\sum_{i=1}^{m} \lambda_{i} y\left(\tau_{i}\right) \\
& =\frac{I_{0^{+}}^{1-\gamma} y\left(0^{+}\right)}{\Gamma(\gamma)} \sum_{i=1}^{m} \lambda_{i} \tau_{i}^{\gamma-1}+\frac{1}{\Gamma(\alpha)} \sum_{i=1}^{m} \lambda_{i} \int_{0}^{\tau_{i}}\left(\tau_{i}-s\right)^{\alpha-1} g(s) d s,
\end{aligned}
$$

which implies

$$
I_{0^{+}}^{1-\gamma} y\left(0^{+}\right)=\frac{\Gamma(\gamma)}{\Gamma(\alpha)\left(\Gamma(\gamma)-\sum_{i=1}^{m} \lambda_{i} \tau_{i}^{\gamma-1}\right)} \sum_{i=1}^{m} \lambda_{i} \int_{0}^{\tau_{i}}\left(\tau_{i}-s\right)^{\alpha-1} g(s) d s
$$


Submitting (3.6) to (3.4), we get for each $t \in(0, T]$

$$
y(t)=w(t)+\frac{1}{\Gamma(\alpha)} \int_{0}^{t}(t-s)^{\alpha-1} g(s) d s,
$$

which is the fixed point of $N$. Now, we prove the sufficiency. Let $y \in C_{1-\gamma}^{\gamma}(J)$ the fixed point of operator $N$, which can be written as (3.7). Applying the operator $D_{0^{+}}^{\gamma}$ to both sides of (3.7), it follows from Lemmas 2.12 and 2.7 that

$$
D_{0^{+}}^{\gamma} y=D_{0^{+}}^{\beta(1-\alpha)} g \text {. }
$$

From (3.8), Definition 2.6 and $D_{0^{+}}^{\gamma} y \in C_{1-\gamma}(J)$, we have

$$
D I_{0^{+}}^{1-\beta(1-\alpha)} g=D_{0^{+}}^{\beta(1-\alpha)} g \in C_{1-\gamma}(J) .
$$

Also, since $g \in C_{1-\gamma}(J)$, by Lemma 2.4, we have

$$
I_{0^{+}}^{1-\beta(1-\alpha)} g \in C(J) .
$$

It follows from $(3.9),(3.10)$ that

$$
I_{0^{+}}^{1-\beta(1-\alpha)} g \in C_{1-\gamma}^{1}(J) .
$$

Thus, $g$ and $I_{0^{+}}^{1-\beta(1-\alpha)} g$ satisfy the conditions of Lemma 2.10.

Now, applying $I_{0^{+}}^{\beta(1-\alpha)}$ to both sides of (3.8) and using Remark 2.11 and Lemma 2.10, we can write

$$
D_{0^{+}}^{\alpha, \beta} y(t)=g(t)-\frac{\left[I_{0^{+}}^{1-\beta(1-\alpha)} g\right](0)}{\Gamma(\beta(1-\alpha))} t^{\beta(1-\alpha)-1} .
$$

Since $1-\gamma<1-\beta(1-\alpha)$, Lemma 2.5 implies that

$$
\left[I_{0^{+}}^{1-\beta(1-\alpha)} g\right](0)=0 .
$$

Hence, the relation (3.11) reduces to

$$
D_{0^{+}}^{\alpha, \beta} y(t)=g(t), \quad t \in(0, T] .
$$

Now, we show that the initial condition (1.2) also holds.

We apply $I_{0^{+}}^{1-\gamma}$ to both sides of (3.7), we have

$$
I_{0^{+}}^{1-\gamma} y(t)=\frac{I_{0^{+}}^{1-\gamma} t^{\gamma-1}}{\Gamma(\alpha)\left(\Gamma(\gamma)-\sum_{i=1}^{m} \lambda_{i} \tau_{i}^{\gamma-1}\right)} \sum_{i=1}^{m} \lambda_{i} \int_{0}^{\tau_{i}}\left(\tau_{i}-s\right)^{\alpha-1} g(s) d s+I_{0^{+}}^{1-\gamma} I_{0^{+}}^{\alpha} g(t)
$$

using the Lemmas 2.10 and 2.11,

$$
I_{0^{+}}^{1-\gamma} y(t)=\frac{\Gamma(\gamma)}{\Gamma(\alpha)\left(\Gamma(\gamma)-\sum_{i=1}^{m} \lambda_{i} \tau_{i}^{\gamma-1}\right)} \sum_{i=1}^{m} \lambda_{i} \int_{0}^{\tau_{i}}\left(\tau_{i}-s\right)^{\alpha-1} g(s) d s+I_{0^{+}}^{1-\beta(1-\alpha)} g(t)
$$


Since $1-\gamma<1-\beta(1-\alpha)$, Lemma 2.5 can be used when taking the limit as $t \rightarrow 0$,

$$
I_{0^{+}}^{1-\gamma} y(0)=\frac{\Gamma(\gamma)}{\Gamma(\alpha)\left(\Gamma(\gamma)-\sum_{i=1}^{m} \lambda_{i} \tau_{i}^{\gamma-1}\right)} \sum_{i=1}^{m} \lambda_{i} \int_{0}^{\tau_{i}}\left(\tau_{i}-s\right)^{\alpha-1} g(s) d s
$$

Substituting $t=\tau_{i}$ into (3.7), we have

$$
\begin{aligned}
y\left(\tau_{i}\right) & =\frac{\tau_{i}^{\gamma-1}}{\Gamma(\alpha)\left(\Gamma(\gamma)-\sum_{i=1}^{m} \lambda_{i} \tau_{i}^{\gamma-1}\right)} \sum_{i=1}^{m} \lambda_{i} \int_{0}^{\tau_{i}}\left(\tau_{i}-s\right)^{\alpha-1} g(s) d s \\
& +\frac{1}{\Gamma(\alpha)} \int_{0}^{\tau_{i}}\left(\tau_{i}-s\right)^{\alpha-1} g(s) d s
\end{aligned}
$$

Then, we derive

$$
\begin{aligned}
\sum_{i=1}^{m} \lambda_{i} y\left(\tau_{i}\right) & =\frac{1}{\Gamma(\alpha)\left(\Gamma(\gamma)-\sum_{i=1}^{m} \lambda_{i} \tau_{i}^{\gamma-1}\right)} \sum_{i=1}^{m} \lambda_{i} \int_{0}^{\tau_{i}}\left(\tau_{i}-s\right)^{\alpha-1} g(s) d s \sum_{i=1}^{m} \lambda_{i} \tau_{i}^{\gamma-1} \\
& +\frac{1}{\Gamma(\alpha)} \sum_{i=1}^{m} \lambda_{i} \int_{0}^{\tau_{i}}\left(\tau_{i}-s\right)^{\alpha-1} g(s) d s \\
& =\frac{1}{\Gamma(\alpha)} \sum_{i=1}^{m} \lambda_{i} \int_{0}^{\tau_{i}}\left(\tau_{i}-s\right)^{\alpha-1} g(s) d s\left(\frac{\sum_{i=1}^{m} \lambda_{i} \tau_{i}^{\gamma-1}}{\Gamma(\gamma)-\sum_{i=1}^{m} \lambda_{i} \tau_{i}^{\gamma-1}}+1\right)
\end{aligned}
$$

Thus

$$
\sum_{i=1}^{m} \lambda_{i} y\left(\tau_{i}\right)=\frac{\Gamma(\gamma)}{\Gamma(\alpha)\left(\Gamma(\gamma)-\sum_{i=1}^{m} \lambda_{i} \tau_{i}^{\gamma-1}\right)} \sum_{i=1}^{m} \lambda_{i} \int_{0}^{\tau_{i}}\left(\tau_{i}-s\right)^{\alpha-1} g(s) d s
$$

It follows (3.12) and (3.13) that

$$
I_{0^{+}}^{1-\gamma} y(0)=\sum_{i=1}^{m} \lambda_{i} y\left(\tau_{i}\right) .
$$

Theorem 3.2. Let the hypotheses

(H1). The function $f:(0, T] \times \mathbb{R} \times \mathbb{R} \rightarrow \mathbb{R}$ such that

$$
f(\cdot, u(\cdot), v(\cdot)) \in C_{1-\gamma}^{\beta(1-\alpha)} \text { for any } u, v \in C_{1-\gamma}(J)
$$

(H2). There exist constants $K>0$ and $0<\bar{K}<1$ such that $|f(t, u, v)-f(t, \bar{u}, \bar{v})| \leq K|u-\bar{u}|+\bar{K}|v-\bar{v}|$ for any $u, v, \bar{u}, \bar{v} \in \mathbb{R} \quad$ and $t \in(0, T]$. 
If

$$
\frac{K \Gamma(\gamma)}{(1-\bar{K}) \Gamma(\alpha+\gamma)}\left[\frac{\sum_{i=1}^{m} \lambda_{i} \tau_{i}^{\alpha+\gamma-1}}{\left|\Gamma(\gamma)-\sum_{i=1}^{m} \lambda_{i} \tau_{i}^{\gamma-1}\right|}+T^{\alpha}\right]<1
$$

then, there exists a unique solution for the Cauchy-type problem (1.1) - (1.2) in the space $C_{1-\gamma}^{\gamma}(J)$.

Proof. The proof will be given in two steps.

Step 1. We show that the operator $N$ defined in (3.1) has a unique fixed point $y^{*}$ in $C_{1-\gamma}(J)$. Let $y, u \in C_{1-\gamma}(J)$ and $t \in(0, T]$, then, we have

$$
\begin{aligned}
|N y(t)-N u(t)| & \leq \frac{t^{\gamma-1}}{\Gamma(\alpha)\left|\Gamma(\gamma)-\sum_{i=1}^{m} \lambda_{i} \tau_{i}^{\gamma-1}\right|} \sum_{i=1}^{m} \lambda_{i} \int_{0}^{\tau_{i}}\left(\tau_{i}-s\right)^{\alpha-1}|g(s)-h(s)| d s \\
& +\frac{1}{\Gamma(\alpha)} \int_{0}^{t}(t-s)^{\alpha-1}|g(s)-h(s)| d s,
\end{aligned}
$$

where $g, h \in C_{1-\gamma}(J)$ such that

$$
\begin{aligned}
& g(t)=f(t, y(t), g(t)) . \\
& h(t)=f(t, u(t), h(t)) .
\end{aligned}
$$

By (H2), we have

$$
\begin{aligned}
|g(t)-h(t)| & =|f(t, y(t), g(t))-f(t, u(t), h(t))| \\
& \leq K|y(t)-u(t)|+\bar{K}|g(t)-h(t)| .
\end{aligned}
$$

Then

$$
|g(t)-h(t)| \leq \frac{K}{1-\bar{K}}|y(t)-u(t)| .
$$

Therefore, for each $t \in(0, T]$

$$
\begin{gathered}
|N y(t)-N u(t)| \leq \frac{K t^{\gamma-1}}{(1-\bar{K}) \Gamma(\alpha)\left|\Gamma(\gamma)-\sum_{i=1}^{m} \lambda_{i} \tau_{i}^{\gamma-1}\right|} \sum_{i=1}^{m} \lambda_{i} \int_{0}^{\tau_{i}}\left(\tau_{i}-s\right)^{\alpha-1}|y(s)-u(s)| d s \\
+\frac{K}{(1-\bar{K}) \Gamma(\alpha)} \int_{0}^{t}(t-s)^{\alpha-1}|y(s)-u(s)| d s \\
=\frac{K t^{\gamma-1}}{(1-\bar{K}) \Gamma(\alpha)\left|\Gamma(\gamma)-\sum_{i=1}^{m} \lambda_{i} \tau_{i}^{\gamma-1}\right|} \sum_{i=1}^{m} \lambda_{i} \int_{0}^{\tau_{i}}\left(\tau_{i}-s\right)^{\alpha-1} s^{\gamma-1}\left|s^{1-\gamma}[y(s)-u(s)]\right| d s \\
+\frac{K}{(1-\bar{K}) \Gamma(\alpha)} \int_{0}^{t}(t-s)^{\alpha-1} s^{\gamma-1}\left|s^{1-\gamma}[y(s)-u(s)]\right| d s
\end{gathered}
$$




$$
\leq \frac{K t^{\gamma-1}\|y-u\|_{C_{1-\gamma}}}{(1-\bar{K})\left|\Gamma(\gamma)-\sum_{i=1}^{m} \lambda_{i} \tau_{i}^{\gamma-1}\right|} \sum_{i=1}^{m} \lambda_{i} I_{0^{+}}^{\alpha}\left(\tau_{i}^{\gamma-1}\right)+\frac{K\|y-u\|_{C_{1-\gamma}}}{1-\bar{K}} I_{0^{+}}^{\alpha}\left(t^{\gamma-1}\right) .
$$

By Lemma 2.7, we have

$$
\begin{aligned}
|N y(t)-N u(t)| & \leq \frac{K \Gamma(\gamma) t^{\gamma-1}\|y-u\|_{C_{1-\gamma}}}{\Gamma(\alpha+\gamma)(1-\bar{K})\left|\Gamma(\gamma)-\sum_{i=1}^{m} \lambda_{i} \tau_{i}^{\gamma-1}\right|} \sum_{i=1}^{m} \lambda_{i} \tau_{i}^{\alpha+\gamma-1} \\
& +\frac{K \Gamma(\gamma) t^{\alpha+\gamma-1}}{(1-\bar{K}) \Gamma(\alpha+\gamma)}\|y-u\|_{C_{1-\gamma}},
\end{aligned}
$$

hence

$$
\begin{aligned}
\left|t^{1-\gamma}(N y(t)-N u(t))\right| \leq \frac{K \Gamma(\gamma)}{(1-\bar{K}) \Gamma(\alpha+\gamma)}\left[\frac{\sum_{i=1}^{m} \lambda_{i} \tau_{i}^{\alpha+\gamma-1}}{\left|\Gamma(\gamma)-\sum_{i=1}^{m} \lambda_{i} \tau_{i}^{\gamma-1}\right|}+t^{\alpha}\right]\|y-u\|_{C_{1-\gamma}} \\
\leq \frac{K \Gamma(\gamma)}{(1-\bar{K}) \Gamma(\alpha+\gamma)}\left[\frac{\sum_{i=1}^{m} \lambda_{i} \tau_{i}^{\alpha+\gamma-1}}{\left|\Gamma(\gamma)-\sum_{i=1}^{m} \lambda_{i} \tau_{i}^{\gamma-1}\right|}+T^{\alpha}\right]\|y-u\|_{C_{1-\gamma}}
\end{aligned}
$$

which implies that

$$
\|N y-N u\|_{C_{1-\gamma}} \leq \frac{K \Gamma(\gamma)}{(1-\bar{K}) \Gamma(\alpha+\gamma)}\left[\frac{\sum_{i=1}^{m} \lambda_{i} \tau_{i}^{\alpha+\gamma-1}}{\left|\Gamma(\gamma)-\sum_{i=1}^{m} \lambda_{i} \tau_{i}^{\gamma-1}\right|}+T^{\alpha}\right]\|y-u\|_{C_{1-\gamma}} .
$$

By (3.14), the operator $N$ is a contraction. Hence, by Banach's contraction principle, $N$ has a unique fixed point $y^{*} \in C_{1-\gamma}(J)$.

Step 2. We show that such a fixed point $y^{*} \in C_{1-\gamma}(J)$ is actually in $C_{1-\gamma}^{\gamma}(J)$.

Since $y^{*}$ is the unique fixed point of operator $N$ in $C_{1-\gamma}(J)$, then, for each $t \in(0, T]$, we have

$$
\begin{aligned}
y^{*}(t) & =w(t)+I_{0^{+}}^{\alpha} g(t) \\
& =\frac{t^{\gamma-1}}{\Gamma(\alpha)\left(\Gamma(\gamma)-\sum_{i=1}^{m} \lambda_{i} \tau_{i}^{\gamma-1}\right)} \sum_{i=1}^{m} \lambda_{i} \int_{0}^{\tau_{i}}\left(\tau_{i}-s\right)^{\alpha-1} f\left(s, y^{*}(s), g(s)\right) d s \\
& +I_{0^{+}}^{\alpha} f\left(t, y^{*}(t), g(t)\right) .
\end{aligned}
$$


Applying $D_{0^{+}}^{\gamma}$ to both sides and by Lemma 2.7, we have

$$
\begin{aligned}
D_{0^{+}}^{\gamma} y^{*}(t) & =D_{0^{+}}^{\gamma}\left[I_{0^{+}}^{\alpha} f\left(t, y^{*}(t), g(t)\right)\right] \\
& =D_{0^{+}}^{\gamma-\alpha} f\left(t, y^{*}(t), g(t)\right) \\
& =D_{0^{+}}^{\beta(1-\alpha)} f\left(t, y^{*}(t), g(t)\right) .
\end{aligned}
$$

Since $\gamma \geq \alpha$, by (H1), the right hand side is in $C_{1-\gamma}(J)$ and thus $D_{0^{+}}^{\gamma} y^{*} \in C_{1-\gamma}(J)$ which implies that $y^{*} \in C_{1-\gamma}^{\gamma}(J)$. As a consequence of Step 1 and 2 together with Theorem 3.1, we can conclude that the problem (1.1)-(1.2) has a unique solution in $C_{1-\gamma}^{\gamma}(J)$.

\section{Ulam-Hyers-Rassias stability}

Theorem 4.1. Assume that (H1), (H2) and (3.14) are satisfied, then the problem (1.1)(1.2) is Ulam-Hyers stable.

Proof. Let $\epsilon>0$ and let $z \in C_{1-\gamma}^{\gamma}(J)$ be a function which satisfies the inequality:

$$
\left|D_{0^{+}}^{\alpha, \beta} z(t)-f\left(t, z(t), D_{0^{+}}^{\alpha, \beta} z(t)\right)\right| \leq \epsilon \quad \text { for any } t \in(0, T]
$$

and let $y \in C_{1-\gamma}^{\gamma}(J)$ be the unique solution of the following Cauchy problem

$$
\begin{gathered}
D_{0^{+}}^{\alpha, \beta} y(t)=f\left(t, y(t), D_{0^{+}}^{\alpha, \beta} y(t)\right), \text { for every } t \in(0, T], T>0, \\
I_{0^{+}}^{1-\gamma} y\left(0^{+}\right)=I_{0^{+}}^{1-\gamma} z\left(0^{+}\right)=\sum_{i=1}^{m} \lambda_{i} y\left(\tau_{i}\right) .
\end{gathered}
$$

Using Theorem 3.1, we obtain

$$
y(t)=\frac{I_{0^{+}}^{1-\gamma} y\left(0^{+}\right)}{\Gamma(\gamma)} t^{\gamma-1}+\frac{1}{\Gamma(\alpha)} \int_{0}^{t}(t-s)^{\alpha-1} g(s) d s, \quad t \in(0, T],
$$

where $g:(0, T] \rightarrow \mathbb{R}$ be a function satisfies the functional equation

$$
g(t)=f(t, y(t), g(t)) .
$$

Now, applying $I_{0^{+}}^{\alpha}$ to both sides of the inequality (4.1), we obtain

$$
\left|I_{0^{+}}^{\alpha} D_{0^{+}}^{\alpha, \beta} z(t)-\frac{1}{\Gamma(\alpha)} \int_{0}^{t}(t-s)^{\alpha-1} h(s) d s\right| \leq \frac{\epsilon t^{\alpha}}{\Gamma(\alpha+1)} \leq \frac{\epsilon T^{\alpha}}{\Gamma(\alpha+1)},
$$

where $h:(0, T] \rightarrow \mathbb{R}$ be a function satisfies the functional equation

$$
h(t)=f(t, z(t), h(t)) .
$$

By the definition of $C_{1-\gamma}^{\gamma}(J)$, Lemma 2.4 and Definition 2.6, we have

$$
I_{0^{+}}^{1-\gamma} z \in C(J) \text { and } D_{0^{+}}^{\gamma} z=D\left(I_{0^{+}}^{1-\gamma} z\right) \in C_{1-\gamma}(J) .
$$

Thus, we have

$$
I_{0^{+}}^{1-\gamma} z \in C_{1-\gamma}^{1}(J)
$$


Now, applying Lemma 2.10 to obtain

$$
I_{0^{+}}^{\gamma} D_{0^{+}}^{\gamma} z(t)=z(t)-\frac{I_{0^{+}}^{1-\gamma} z\left(0^{+}\right)}{\Gamma(\gamma)} t^{\gamma-1}, \quad t \in(0, T] .
$$

Since $D_{0^{+}}^{\gamma} z \in C_{1-\gamma}(J)$, Lemma 2.12 yields

$$
\left(I_{0^{+}}^{\gamma} D_{0^{+}}^{\gamma} z\right)(t)=\left(I_{0^{+}}^{\alpha} D_{0^{+}}^{\alpha, \beta} z\right)(t), \quad t \in(0, T] .
$$

From (4.3) and (4.4), we get

$$
\left(I_{0^{+}}^{\alpha} D_{0^{+}}^{\alpha, \beta} z\right)(t)=z(t)-\frac{I_{0^{+}}^{1-\gamma} z\left(0^{+}\right)}{\Gamma(\gamma)} t^{\gamma-1}, \quad t \in(0, T]
$$

By replacing (4.5) in (4.2), we have

$$
\left|z(t)-\frac{I_{0^{+}}^{1-\gamma} z\left(0^{+}\right)}{\Gamma(\gamma)} t^{\gamma-1}-\frac{1}{\Gamma(\alpha)} \int_{0}^{t}(t-s)^{\alpha-1} h(s) d s\right| \leq \frac{\epsilon T^{\alpha}}{\Gamma(\alpha+1)} .
$$

We have for any $t \in(0, T]$

$$
\begin{aligned}
|z(t)-y(t)|= & \mid z(t)-\frac{I_{0^{+}}^{1-\gamma} y\left(0^{+}\right)}{\Gamma(\gamma)} t^{\gamma-1}-\frac{1}{\Gamma(\alpha)} \int_{0}^{t}(t-s)^{\alpha-1} h(s) d s \\
& +\frac{1}{\Gamma(\alpha)} \int_{0}^{t}(t-s)^{\alpha-1}(h(s)-g(s)) d s \mid \\
\leq & \left|z(t)-\frac{I_{0^{+}}^{1-\gamma} z\left(0^{+}\right)}{\Gamma(\gamma)} t^{\gamma-1}-\frac{1}{\Gamma(\alpha)} \int_{0}^{t}(t-s)^{\alpha-1} h(s) d s\right| \\
& +\frac{1}{\Gamma(\alpha)} \int_{0}^{t}(t-s)^{\alpha-1}|h(s)-g(s)| d s .
\end{aligned}
$$

Thus

$$
|z(t)-y(t)| \leq \frac{\epsilon T^{\alpha}}{\Gamma(\alpha+1)}+\frac{1}{\Gamma(\alpha)} \int_{0}^{t}(t-s)^{\alpha-1}|h(s)-g(s)| d s, \quad t \in(0, T] .
$$

By (H2), we have for each $t \in(0, T]$

$$
\begin{aligned}
|h(t)-g(t)| & =|f(t, z(t), h(t))-f(t, y(t), g(t))| \\
& \leq K|z(t)-y(t)|+\bar{K}|h(t)-g(t)| .
\end{aligned}
$$

Then

$$
|h(t)-g(t)| \leq \frac{K}{1-\bar{K}}|z(t)-y(t)| .
$$

Using (4.6) and (4.7), we obtain

$$
|z(t)-y(t)| \leq \frac{\epsilon T^{\alpha}}{\Gamma(\alpha+1)}+\frac{K}{(1-\bar{K}) \Gamma(\alpha)} \int_{0}^{t}(t-s)^{\alpha-1}|z(s)-y(s)| d s, \quad t \in(0, T] .
$$

By Lemma 2.15, we have

$$
|z(t)-y(t)| \leq \frac{\epsilon T^{\alpha}}{\Gamma(\alpha+1)}\left[1+\frac{\delta K T^{\alpha}}{(1-\bar{K}) \Gamma(\alpha+1)}\right]:=c \epsilon
$$


where $\delta=\delta(\alpha)$ a constant, which completes the proof of the theorem. Moreover, if we set $\psi(\epsilon)=c \epsilon ; \psi(0)=0$, then the problem (1.1)-(1.2) is generalized Ulam-Hyers stable.

Theorem 4.2. Assume that (H1), (H2), (3.14) and

(H3) there exists an increasing function $\varphi \in C\left(J, \mathbb{R}_{+}\right)$and there exists $\lambda_{\varphi}>0$ such that for any $t \in(0, T]$

$$
I_{0^{+}}^{\alpha} \varphi(t) \leq \lambda_{\varphi} \varphi(t)
$$

are satisfied, then, the problem (1.1)-(1.2) is Ulam-Hyers-Rassias stable.

Proof. Let $z \in C_{1-\gamma}^{\gamma}(J)$ be a function which satisfies the inequality:

$$
\left|D_{0^{+}}^{\alpha, \beta} z(t)-f\left(t, z(t), D_{0^{+}}^{\alpha, \beta} z(t)\right)\right| \leq \epsilon \varphi(t) \quad \text { for any } t \in(0, T], \epsilon>0
$$

and let $y \in C_{1-\gamma}^{\gamma}(J)$ be the unique solution of the following Cauchy problem

$$
\begin{gathered}
D_{0^{+}}^{\alpha, \beta} y(t)=f\left(t, y(t), D_{0^{+}}^{\alpha, \beta} y(t)\right), \text { for every } t \in(0, T], T>0, \\
I_{0^{+}}^{1-\gamma} y\left(0^{+}\right)=I_{0^{+}}^{1-\gamma} z\left(0^{+}\right)=\sum_{i=1}^{m} \lambda_{i} y\left(\tau_{i}\right) .
\end{gathered}
$$

Using Theorem 3.1, we obtain

$$
y(t)=\frac{I_{0^{+}}^{1-\gamma} y\left(0^{+}\right)}{\Gamma(\gamma)} t^{\gamma-1}+\frac{1}{\Gamma(\alpha)} \int_{0}^{t}(t-s)^{\alpha-1} g(s) d s, \quad t \in(0, T],
$$

where $g:(0, T] \rightarrow \mathbb{R}$ be a function satisfies the functional equation

$$
g(t)=f(t, y(t), g(t)) .
$$

Now, applying $I_{0^{+}}^{\alpha}$ to both sides of the inequality (4.8), we obtain

$$
\left|I_{0^{+}}^{\alpha} D_{0^{+}}^{\alpha, \beta} z(t)-\frac{1}{\Gamma(\alpha)} \int_{0}^{t}(t-s)^{\alpha-1} h(s) d s\right| \leq \frac{\epsilon}{\Gamma(\alpha)} \int_{0}^{t}(t-s)^{\alpha-1} \varphi(s) d s,
$$

where $h:(0, T] \rightarrow \mathbb{R}$ be a function satisfies the functional equation

$$
h(t)=f(t, z(t), h(t)) .
$$

Using (H3), we have for each $t \in(0, T]$

$$
\left|I_{0^{+}}^{\alpha} D_{0^{+}}^{\alpha, \beta} z(t)-\frac{1}{\Gamma(\alpha)} \int_{0}^{t}(t-s)^{\alpha-1} h(s) d s\right| \leq \epsilon \lambda_{\varphi} \varphi(t) .
$$

From the proof of Theorem 4.1, we obtain

$$
\left|z(t)-\frac{I_{0^{+}}^{1-\gamma} z\left(0^{+}\right)}{\Gamma(\gamma)} t^{\gamma-1}-\frac{1}{\Gamma(\alpha)} \int_{0}^{t}(t-s)^{\alpha-1} h(s) d s\right| \leq \epsilon \lambda_{\varphi} \varphi(t) .
$$


We have for any $t \in(0, T]$

$$
\begin{aligned}
|z(t)-y(t)|= & \mid z(t)-\frac{I_{0^{+}}^{1-\gamma} y\left(0^{+}\right)}{\Gamma(\gamma)} t^{\gamma-1}-\frac{1}{\Gamma(\alpha)} \int_{0}^{t}(t-s)^{\alpha-1} h(s) d s \\
& +\frac{1}{\Gamma(\alpha)} \int_{0}^{t}(t-s)^{\alpha-1}[h(s)-g(s)] d s \mid \\
\leq & \left|z(t)-\frac{I_{0^{+}}^{1-\gamma} z\left(0^{+}\right)}{\Gamma(\gamma)} t^{\gamma-1}-\frac{1}{\Gamma(\alpha)} \int_{0}^{t}(t-s)^{\alpha-1} h(s) d s\right| \\
& +\frac{1}{\Gamma(\alpha)} \int_{0}^{t}(t-s)^{\alpha-1}|h(s)-g(s)| d s .
\end{aligned}
$$

Thus

$$
|z(t)-y(t)| \leq \epsilon \lambda_{\varphi} \varphi(t)+\frac{1}{\Gamma(\alpha)} \int_{0}^{t}(t-s)^{\alpha-1}|h(s)-g(s)| d s, \quad t \in(0, T] .
$$

By (H2), we have for each $t \in(0, T]$

$$
\begin{aligned}
|h(t)-g(t)| & =|f(t, z(t), h(t))-f(t, y(t), g(t))| \\
& \leq K|z(t)-y(t)|+\bar{K}|h(t)-g(t)|,
\end{aligned}
$$

then

$$
|h(t)-g(t)| \leq \frac{K}{1-\bar{K}}|z(t)-y(t)| .
$$

Using (4.9) and (4.10), we have

$$
|z(t)-y(t)| \leq \epsilon \lambda_{\varphi} \varphi(t)+\frac{K}{(1-\bar{K}) \Gamma(\alpha)} \int_{0}^{t}(t-s)^{\alpha-1}|z(s)-y(s)| d s, \quad t \in(0, T] .
$$

By Lemma 2.15, we obtain

$$
|z(t)-y(t)| \leq \epsilon \lambda_{\varphi} \varphi(t)+\frac{\delta_{1} \epsilon K \lambda_{\varphi}}{(1-\bar{K}) \Gamma(\alpha)} \int_{0}^{t}(t-s)^{\alpha-1} \varphi(s) d s
$$

where $\delta_{1}=\delta_{1}(\alpha)$ is constant, and by $\left(H_{2}\right)$, we have

$$
|z(t)-y(t)| \leq \epsilon \lambda_{\varphi} \varphi(t)+\frac{\delta_{1} \epsilon K \lambda_{\varphi}^{2} \varphi(t)}{1-\bar{K}}=\left(1+\frac{\delta_{1} K \lambda_{\varphi}}{1-\bar{K}}\right) \epsilon \lambda_{\varphi} \varphi(t) .
$$

Then, for any $t \in(0, T]$

$$
|z(t)-y(t)| \leq\left[\left(1+\frac{\delta_{1} K \lambda_{\varphi}}{1-\bar{K}}\right) \lambda_{\varphi}\right] \epsilon \varphi(t)=\operatorname{c\epsilon \varphi }(t)
$$

which completes the proof of Theorem 4.2. 


\section{Examples}

Example 5.1. Consider the following problem of non-linear implicit fractional differential equations

$$
\begin{gathered}
D_{0^{+}}^{\frac{1}{2}, 0} y(t)=\frac{1}{10 e^{-t+2}\left(1+|y(t)|+\left|D_{0^{+}}^{\frac{1}{2}, 0} y(t)\right|\right)}+\frac{1}{\sqrt{t}} \text { for each } t \in(0,1] \\
I_{0^{+}}^{\frac{1}{2}} y\left(0^{+}\right)=3 y\left(\frac{1}{3}\right)+2 y\left(\frac{1}{2}\right) .
\end{gathered}
$$

Set

We have

$$
f(t, u, v)=\frac{1}{10 e^{-t+2}(1+|u|+|v|)}+\frac{1}{\sqrt{t}}, \quad t \in(0,1], \quad u, v \in \mathbb{R} .
$$

$$
C_{1-\gamma}^{\beta(1-\alpha)}([0,1])=C_{\frac{1}{2}}^{0}([0,1])=\left\{h:(0,1] \rightarrow \mathbb{R}: t^{\frac{1}{2}} h \in C([0,1])\right\},
$$

with $\gamma=\alpha=\frac{1}{2}$ and $\beta=0$. Clearly, the function $f \in C_{\frac{1}{2}}([0,1])$.

Hence condition (H1) is satisfied.

For each $u, \bar{u}, v, \bar{v} \in \mathbb{R}$ and $t \in(0,1]$, we have

$$
|f(t, u, v)-f(t, \bar{u}, \bar{v})| \leq \frac{1}{10 e}(|u-\bar{u}|+|v-\bar{v}|) .
$$

Hence condition (H2) is satisfied with $K=\bar{K}=\frac{1}{10 e}$.

The condition

$$
\frac{K \Gamma(\gamma)}{(1-\bar{K}) \Gamma(\alpha+\gamma)}\left[\frac{\sum_{i=1}^{2} \lambda_{i} \tau_{i}^{\alpha+\gamma-1}}{\left|\Gamma(\gamma)-\sum_{i=1}^{2} \lambda_{i} \tau_{i}^{\gamma-1}\right|}+T^{\alpha}\right] \approx 0.122<1
$$

is satisfied with $\lambda_{1}=3, \lambda_{2}=2, \tau_{1}=\frac{1}{3}, \tau_{2}=\frac{1}{2}$ and $T=1$. It follows from Theorem 3.2 that the problem $(5.1)-(5.2)$ has a unique solution in the space $C_{\frac{1}{2}}^{\frac{1}{2}}([0,1])$. Moreover, Theorem 4.1, implies that the problem (5.1)-(5.2) is Ulam-Hyers stable.

Example 5.2. Consider the following initial value problem

$$
\begin{gathered}
D_{0^{+}}^{\frac{1}{2}, 0} y(t)=\frac{1}{9+e^{-t}}\left[\frac{|y(t)|}{1+|y(t)|}-\frac{\left|D_{0^{+}}^{\frac{1}{2}, 0} y(t)\right|}{1+\left|D_{0^{+}}^{\frac{1}{2}, 0} y(t)\right|}\right]+\frac{t+1}{\sqrt{t}}, t \in(0,1] \\
I_{0^{+}}^{\frac{1}{2}} y\left(0^{+}\right)=2 y\left(\frac{1}{2}\right) .
\end{gathered}
$$

Set

$$
f(t, u, v)=\frac{1}{9+e^{-t}}\left[\frac{u}{1+u}-\frac{v}{1+v}\right]+\frac{t+1}{\sqrt{t}}, t \in(0,1], u, v \in[0,+\infty)
$$


We have

$$
C_{1-\gamma}^{\beta(1-\alpha)}([0,1])=C_{\frac{1}{2}}^{0}([0,1])=\left\{h:(0,1] \rightarrow \mathbb{R}: t^{\frac{1}{2}} h \in C([0,1])\right\},
$$

with $\gamma=\alpha=\frac{1}{2}$ and $\beta=0$. Clearly, the function $f \in C_{\frac{1}{2}}([0,1])$.

Hence condition (H1) is satisfied.

For each $u, \bar{u}, v, \bar{v} \in \mathbb{R}$ and $t \in(0,1]:$

$$
\begin{aligned}
|f(t, u, v)-f(t, \bar{u}, \bar{v})| & \leq \frac{1}{9+e^{-t}}(|u-\bar{u}|+|v-\bar{v}|) \\
& \leq \frac{1}{9+e^{-1}}(|u-\bar{u}|+|v-\bar{v}|)
\end{aligned}
$$

Hence condition $(H 2)$ is satisfied with $K=\bar{K}=\frac{1}{9+e^{-1}}$.

The condition

$$
\frac{K \Gamma(\gamma)}{(1-\bar{K}) \Gamma(\alpha+\gamma)}\left[\frac{\lambda_{1} \tau_{1}^{\alpha+\gamma-1}}{\left|\Gamma(\gamma)-\lambda_{1} \tau_{1}^{\gamma-1}\right|}+T^{\alpha}\right] \approx 0.6077<1
$$

is satisfied with $\lambda_{1}=2, \tau_{1}=\frac{1}{2}$ and $T=1$. It follows from Theorem 3.2 that the problem (5.3)-(5.4) has a unique solution in the space $C_{\frac{1}{2}}^{\frac{1}{2}}([0,1])$, and by Theorem 4.1 , the problem (5.3)-(5.4) is Ulam-Hyers stable.

\section{References}

[1] Abbas, S., Benchohra, M., N'Guérékata, G.M., Topics in Fractional Differential Equations, Springer, New York, 2012.

[2] Abbas, S., Benchohra, M., N'Guérékata, G.M., Advanced Fractional Differential and Integral Equations, Nova Science Publishers, New York, 2015.

[3] Benchohra, M., Lazreg, J.E., Existence and Ulam stability for nonlinear implicit fractional differential equations with Hadamard derivative, Stud. Univ. Babeş-Bolyai Math., 62(2017), no. 1, 27-38.

[4] Furati, K.M., Kassim, M.D., Tatar, N.-E., Existence and uniqueness for a problem involving Hilfer fractional derivative, Comput. Math. Appl., 64(2012), 1616-1626.

[5] Furati, K.M., Kassim, M.D., Tatar, N.-E., Non-existence of global solutions for a differential equation involving Hilfer fractional derivative, Electron. J. Differential Equations, 235(2013), 10 pp.

[6] Gerolymatou, E., Vardoulakis, I., Hilfer, R., Modelling infiltration by means of a nonlinear fractional diffusion model, J. Physics D, Applied Physics, 39(2006), 4104-4110.

[7] Hilfer, R., Applications of Fractional Calculus in Physics, World Scientific, Singapore, 2000.

[8] Hilfer, R., Experimental evidence for fractional time evolution in glass forming materials, Chem. Phys., 284(2002), 399-408.

[9] Hilfer, R., Anton, L., Fractional master equations and fractal time random walks, Physical Review E, 51(1995), 848-851. 
[10] Kilbas, A.A., Srivastava, H.M., Trujillo, J.J., Theory and Applications of Fractional Differential Equations, North-Holland Mathematics Studies, 204, Elsevier Science B.V., Amsterdam, 2006.

[11] Kou, C., Liu, J., Ye, Y., Existence and uniqueness of solutions for the Cauchy-type problems of fractional differential equations, Discrete Dyn. Nat. Soc., 2010(2010), 1-15.

[12] Mainardi, F., Gorenflo, R., Time-fractional derivatives in relaxation processes: a tutorial survey, Fract. Calc. Appl. Anal., 10(2007), no. 3, 269-308.

[13] Podlubny, I., Fractional Differential Equations, in: Mathematics in Science and Engineering, vol. 198, Acad. Press, 1999.

[14] Rus, I.A., Ulam stabilities of ordinary differential equations in a Banach space, Carpathian J. Math., 26(2010), 103-107.

[15] Tomovski, Z., Sandev, T., Metzler, R., Dubbeldam, J., Generalized space-time fractional diffusion equation with composite fractional time derivative, Phys. A, 391(2012), 25272542 .

[16] Tan Wenchang, X.M., Pan, W., A note on unsteady flows of a viscoelastic fluid with the fractional Maxwell model between two parallel plates, Intern. J. Nonlin. Mech., 38(2003), 645-650.

[17] Samko, S.G., Kilbas, A.A., Marichev, O.I., Fractional Integrals and Derivatives, Theory and Applications, Gordon and Breach, Amsterdam, 1987, Engl. Trans. from the Russian.

[18] Sandev, T., Metzler, R., Tomovski, Z., Fractional diffusion equation with a generalized Riemann-Liouville time fractional derivative, J. Phys. A, Math. Theor., 44(2011), 255203.

[19] Wang, J., Zhang, Y., Nonlocal initial value problems for differential equations with Hilfer fractional derivative, Appl. Math. Comput., 266(2015), 850-859.

[20] Ye, H., Gao, J., Ding, Y., A generalized Gronwall inequality and its application to a fractional differential equation, J. Math. Anal. Appl., 328(2007), 1075-1081.

[21] Zhou, Y., Basic Theory of Fractional Differential Equations, World Scientific, Singapore, 2014.

Mouffak Benchohra

Laboratory of Mathematics, Djillali Liabes University of Sidi Bel-Abbes

P.O. Box 89 Sidi Bel Abbes 22000, Algeria

e-mail: benchohra@univ-sba.dz

Soufyane Bouriah

Laboratory of Mathematics, Djillali Liabes University of Sidi Bel-Abbes

P.O. Box 89 Sidi Bel Abbes 22000, Algeria

and

Department of Mathematics, Faculty of Exact Sciences and Informatics

Hassiba Benbouali University, P.O. Box 151 Chlef 02000, Algeria

e-mail: s.bouriah@univ-chlef.dz Bouriahsoufiane@yahoo.fr

Juan J. Nieto

Departamento de Análisis Matemático, Facultad de Matemáticas

Universidad de Santiago de Compostela

Santiago de Compostela, Spain

e-mail: juanjose.nieto.roig@usc.es 